\title{
Letter \\ One-Way Zero Reflection in an Insulator-Metal-Insulator Structure Using the Transfer Matrix Method
}

\author{
Heeso Noh ${ }^{1}\left(\mathbb{D}\right.$ and Jai-Min Choi ${ }^{2, *(\mathbb{D}}$ \\ 1 Department of Physics, Kookmin University, Seoul 02707, Korea; heesonoh@kookmin.ac.kr \\ 2 Division of Science Education, Jeonbuk National University, Jeonju 54896, Korea \\ * Correspondence: jaiminchoi@jbnu.ac.kr; Tel.: +82-63-270-2777
}

check for updates

Citation: Noh, H.; Choi, J.-M. One-Way Zero Reflection in an Insulator-Metal-Insulator Structure Using the Transfer Matrix Method. Photonics 2021, 8, 8. https://doi.org/ $10.3390 /$ photonics 8010008

Received: 11 December 2020 Accepted: 29 December 2020 Published: 31 December 2020

Publisher's Note: MDPI stays neutral with regard to jurisdictional clai$\mathrm{ms}$ in published maps and institutional affiliations.

Copyright: (C) 2020 by the authors. Licensee MDPI, Basel, Switzerland. This article is an open access article distributed under the terms and conditions of the Creative Commons Attribution (CC BY) license (https:// creativecommons.org/licenses/by/ $4.0 /)$.

\begin{abstract}
We numerically demonstrate one-way zero reflection using the transfer matrix method. Using simulations, we adjusted the thickness of $\mathrm{SiO}_{2}$ layers in a simple $\mathrm{SiO}_{2}-\mathrm{Au}-\mathrm{SiO}_{2}$ layer structure. We found two solutions, $47 \mathrm{~nm}-10 \mathrm{~nm}-32 \mathrm{~nm}$ and $71 \mathrm{~nm}-10 \mathrm{~nm}-60 \mathrm{~nm}$, which are the thicknesses for one-way zero reflection at a wavelength of $560 \mathrm{~nm}$. We confirmed it with reflection spectra, where reflectance is zero for forwardly incident light and $2.5 \%$ for backwardly incident light at the wavelength $560 \mathrm{~nm}$, and thickness $47 \mathrm{~nm}-10 \mathrm{~nm}-32 \mathrm{~nm}$.
\end{abstract}

Keywords: reflection; absorption; PT-symmetry

\section{Introduction}

Many physical systems have a Hamiltonian with Hermitian properties, these properties lead to real and orthogonal eigenvalues. Recently Hamiltonians with parity-time (PT) symmetry have attracted a lot of interest because their eigenvalues are real below a certain threshold but are complex above that threshold [1]. PT-symmetric systems have a potential $V(\mathbf{r})=V^{*}(-\mathbf{r})$. Such a system can also be realized in optics [2,3]. If the permittivity, $\varepsilon$, of the system has symmetry such that $\varepsilon(\mathbf{r})=\varepsilon^{*}(-\mathbf{r})$, then PT-symmetry can be realized in an optical systems. Therefore, in order to satisfy PT-symmetry in optics, one should consider optical gain and loss in the system which, in turn, means $\varepsilon$ should be represented as a complex number. This has been demonstrated many times in previous research [4-7].

One of the interesting phenomena in systems with PT-symmetry is the existence of an exceptional point. Exceptional point exists at the threshold where an eigenvalue changes from a real number to a complex number or vice versa. This behavior seems to be similar to that seen with degenerate states. The eigen modes, however, coalesce at an exceptional point. In other words, only one eigen mode can exist, which opens up the possibility of one-way reflection. There has been lots of research that has used this exceptional point in a variety of applications such as in single mode lasers or unidirectional reflectionlessness among others [8-13]. Recent progress in developing exceptional point-based research is well introduced in Ref. [14,15] and references therein.

Even though many physical systems use Hamiltonians to describe the physics at play, this is not the only way to describe PT-symmetry. Any kind of system can be considered, all that is required is that the PT-symmetry condition is satisfied. In optics, a scattering matrix (s-matrix) is often used to describe optical phenomena. Therefore, an s-matrix is also a candidate to describe PT-symmetric systems. One-way zero reflection has been obtained using an s-matrix in previous research [16-18]. Even though the theory for zero reflection comes from the use of an s-matrix, the reflection coefficient can be calculated with other methods. For layered structures, it is convenient to use the transfer matrix method (TMM) [19].

In this report, we use a three-layered structure that is composed of a metal layer sandwiched between two dielectric layers to obtain one-way zero reflection. We selected $\mathrm{Au}$ as a metal layer and $\mathrm{SiO}_{2}$ as dielectric layers which are favored materials in photonics 
research. Although there are demonstrations using Si-based structure [16,17], one-way zero reflection using the metal-based layer structure may also find its applications in various fields. By adjusting the thickness of the $\mathrm{SiO}_{2}$ layers, we obtain zero reflection for forwardly incident light while we get a nonzero reflection value for backwardly incident light.

\section{Simulation}

Reflection and transmission were obtained using TMM for the layered structure. The electric field relation between the $m$ th layer and the $(m+1)$ th layer in the TMM is as follows.

$$
\begin{aligned}
& \left(\begin{array}{c}
E_{m}^{f} \\
E_{m}^{b}
\end{array}\right)=M_{m}\left(\begin{array}{c}
E_{m+1}^{f} \\
E_{m+1}^{b}
\end{array}\right), \\
& M_{m}=\left(\begin{array}{cc}
e^{-i n_{m} k d_{m}} & 0 \\
0 & e^{i n_{m} k d_{m}}
\end{array}\right)\left(\begin{array}{cc}
1 / t_{m, m+1} & r_{m, m+1} / t_{m, m+1} \\
r_{m, m+1} / t_{m, m+1} & 1 / t_{m, m+1}
\end{array}\right),
\end{aligned}
$$

where $E_{m}^{f}\left(E_{m}^{b}\right)$ is the electric field of forwardly (backwardly) propagating light; $n_{m}$ and $d_{m}$ are the refractive index and thickness of the $m$ th layer, respectly; $k$ is the wave number in a vacuum; $r_{m, m+1}$ and $t_{m, m+1}$ are the reflection and transmission coefficients when light propagates from the $m$ th layer to the $(m+1)$ th layer. For normal incidence, the equations for $r_{m, m+1}$ and $t_{m, m+1}$ follow when relative permittivity is 1 .

$$
r_{m, m+1}=\frac{n_{m}-n_{m+1}}{n_{m}+n_{m+1}}, \quad t_{m, m+1}=\frac{2 n_{m}}{n_{m}+n_{m+1}},
$$

where $n_{m+1}$ is the refractive index of the $(m+1)$ th layer.

If the total number of layers is $N$ and the electric field amplitude of incident light is 1 , when the propagation direction of incident light is forward, we can write a matrix form of TMM as follows,

$$
\left(\begin{array}{c}
1 \\
r_{f}
\end{array}\right)=\left(\begin{array}{cc}
1 / t_{0,1} & r_{0,1} / t_{0,1} \\
r_{0,1} / t_{0,1} & 1 / t_{0,1}
\end{array}\right) M_{1} M_{2} \cdots M_{N}\left(\begin{array}{c}
t_{f} \\
0
\end{array}\right)
$$

where $r_{f}, t_{f}$ are the total reflection and transmission coefficients when the propagation direction of incident light is forward. When the propagation direction of incident light is backward, the relation is as follows,

$$
\left(\begin{array}{c}
0 \\
t_{b}
\end{array}\right)=\left(\begin{array}{cc}
1 / t_{0,1} & r_{0,1} / t_{0,1} \\
r_{0,1} / t_{0,1} & 1 / t_{0,1}
\end{array}\right) M_{1} M_{2} \cdots M_{N}\left(\begin{array}{c}
r_{b} \\
1
\end{array}\right) .
$$

In two port system, s-matrix can be expressed as $s=\left(\begin{array}{cc}t & r_{b} \\ r_{f} & t\end{array}\right)$. Because PTsymmetric system maintains reciprocity, $t=t_{f}=t_{b}$. Furthermore, corresponding eignevalues are $s_{n}=t \pm \sqrt{r_{f} r_{b}}$. When either $r_{f}$ or $r_{b}$ is zero, two eigenvalues coalesce to form exceptional points, i.e., the system has one-way zero reflection.

The structure for one-way zero reflection is depicted in Figure 1a. The structure presented consists of $\mathrm{SiO}_{2}-\mathrm{Au}-\mathrm{SiO}_{2}$ layers in vacuum. The thickness of the gold is fixed at $10 \mathrm{~nm}$. The reason for this is that $\mathrm{Au}$ is highly reflective in the visible wavelength range. If the thickness of $\mathrm{Au}$ is too high, we cannot achieve coupling between the light in the top $\mathrm{SiO}_{2}$ layer and that in the bottom $\mathrm{SiO}_{2}$ layer. On the other hand, if the thickness of the $\mathrm{Au}$ is too low, we will no longer be able to achieve a continuous film with the Au. The Au of $10 \mathrm{~nm}$ thickness is right above the percolation threshold [20]. The thicknesses of the top and bottom $\mathrm{SiO}_{2}$ layers are denoted by $d_{1}$ and $d_{2}$, respectively. We adjusted $d_{1}$ and $d_{2}$ to achieve one-way zero reflection. The incident light from the left is denoted as forwardly incident light; the incident light from the right, backwardly incident light. 
(a)

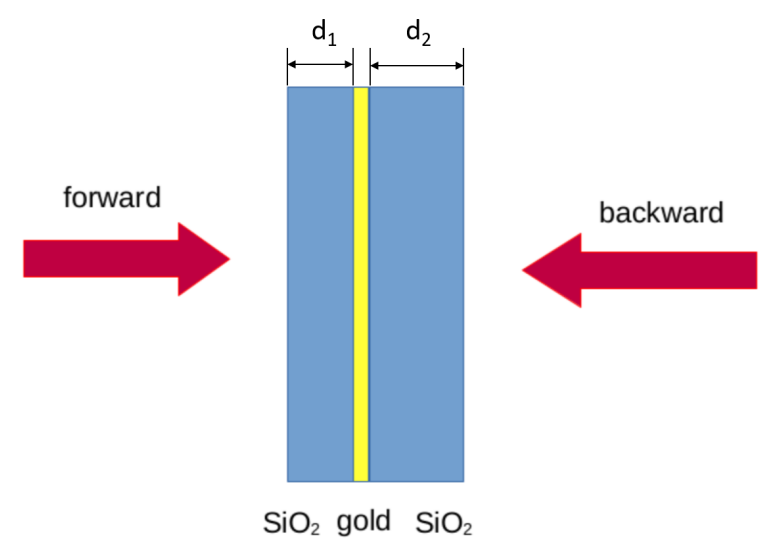

(b)

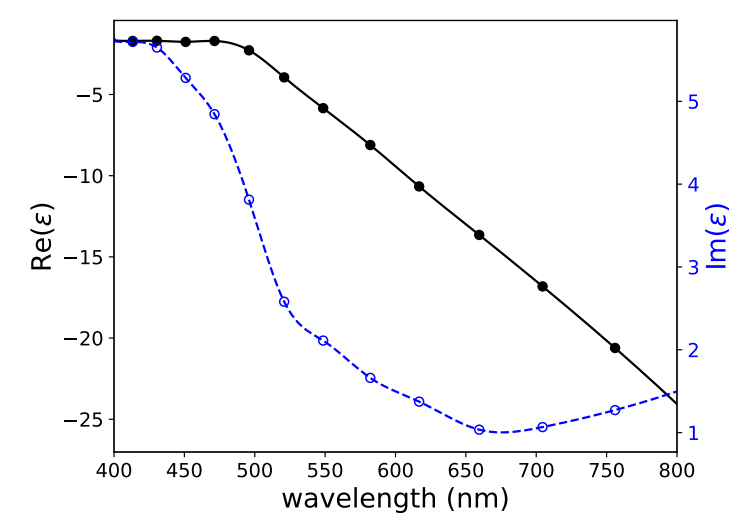

Figure 1. (a) Schematic of simulated structure. The three layers seen here are $\mathrm{SiO}_{2}-\mathrm{Au}-\mathrm{SiO}_{2}$. We fixed the thickness of Au as $10 \mathrm{~nm}$, and $d_{1}$ and $d_{2}$ are varied to achieve one-way zero reflection. Incident light coming from the left (right) is denoted as forwardly (backwardly) incident light. (b) dispersion curve of Au. The data points marked with the black filled circles $(\bullet)$ and the blue open circles (०) are from Ref. [21]. Solid and dashed curves are lines of best fit. The black curves represents the real part of $\varepsilon$, the blue curves represents the imaginary part of $\varepsilon$.

The relative permittivity, $\varepsilon$, is defined as $\varepsilon=\sqrt{n}$. The $\varepsilon$ of $\mathrm{SiO}_{2}$ is 2.13. As the permittivity of $\mathrm{Au}$ is strongly dispersive, its value changing with wavelength is not negligible so one fixed value cannot be used for a range of wavelengths. We used $\varepsilon$ of Au from Ref. [21]. Figure $1 \mathrm{~b}$ shows the dispersion of $\varepsilon$ as a function of wavelength. Blue represents the real part of $\varepsilon$; black represents the imaginary part of $\varepsilon$. The data points marked with the filled circles $(\bullet)$ and the open circles (०) come from the reference, while the solid and dashed curves come from interpolation with the spline method. The real part of $\varepsilon$ is negative because $\mathrm{Au}$ is a metal.

$r_{f}$ and $r_{b}$ are generally represented with complex numbers. Therefore, one can write $r_{f}$ and $r_{b}$ in terms of amplitude and phase. Using phase gives a great advantage when one is trying to find a zero for $r_{f}$. As such, it is possible to find where $r_{f}=0$ by simply observing the phase of $r_{f}$ because there will be a $2 \pi$ phase change around the value zero [22]. We continuously adjusted $d_{1}$ and $d_{2}$ from $20 \mathrm{~nm}$ to $100 \mathrm{~nm}$ to find the phases of $r_{f}$ and $r_{b}$.

Figures 2a,b show the false color images of the phases of $r_{f}$ and $r_{b}$ respectively, when the wavelength $\lambda$ of the incident light is $560 \mathrm{~nm}$. The colors indicate the phases of $r_{f}$ and $r_{b}$. One can see that the phase changes by $2 \pi$, i.e., the color changes from the darkest blue to the darkest red, if we move counterclockwise around the bottom-left point $\mathbf{P}_{1}=(47 \mathrm{~nm}, 32 \mathrm{~nm})$ of the dark line and around the top-right point $\mathbf{P}_{2}=(71 \mathrm{~nm}, 60 \mathrm{~nm})$ of the dark line, where the first and second numbers correspond to $d_{1}$ and $d_{2}$, respectively. Therefore, we can see that $r_{f}$ becomes zero at $\mathbf{P}_{1}$ and $\mathbf{P}_{2} . r_{b}$, however, does not go to zero at $\mathbf{P}_{1}$ and $\mathbf{P}_{2}$ because there is no $2 \pi$ phase change around those points in false color image in Figure $2 b$. This indicates that one-way zero reflection has been realized at these points. It is also possible to get more accurate values for $d_{1}$ and $d_{2}$ by increasing the resolution of the false color image; this can be done by decreasing the scanning step, i.e., by reducing the interval we increment $d_{1}$ and $d_{2}$ while creating these false color image. Note that $r_{b}$ goes to zero at $\mathbf{P}_{1}^{\mathrm{T}}=(32 \mathrm{~nm}, 47 \mathrm{~nm})$ and $\mathbf{P}_{2}^{\mathrm{T}}=(60 \mathrm{~nm}, 71 \mathrm{~nm})$, where $\mathbf{P}_{1}^{\mathrm{T}}\left(\mathbf{P}_{2}^{\mathrm{T}}\right)$ is transposed of $\mathbf{P}_{1}\left(\mathbf{P}_{2}\right)$. This is because the Au layer is embedded with the two $\mathrm{SiO}_{2}$ layers. 
(a)

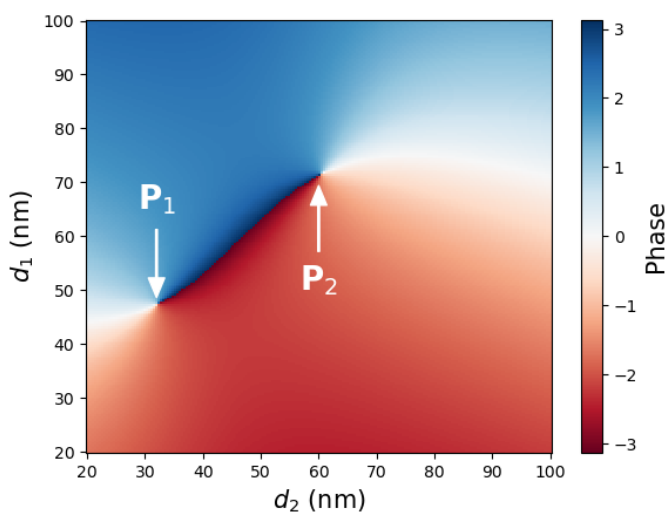

(b)

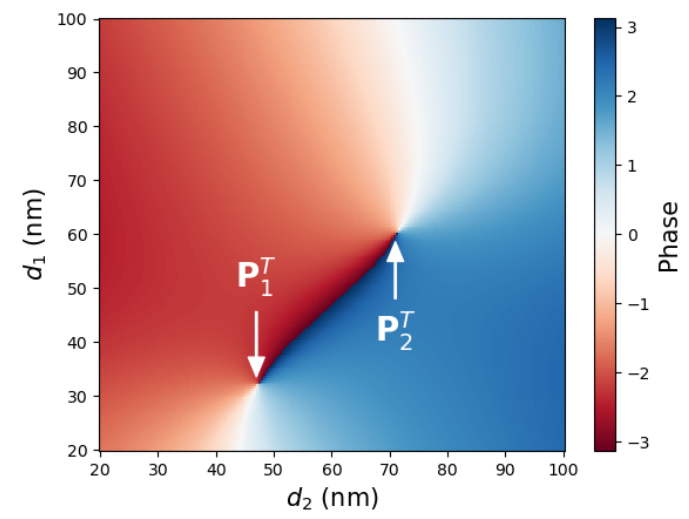

Figure 2. The false color image showing the phases of $r_{f}(\mathrm{a})$ and $r_{b}(\mathrm{~b})$. (a) $r_{f}=0$ at $\mathbf{P}_{1}=(47 \mathrm{~nm}, 32 \mathrm{~nm})$ and $\mathbf{P}_{2}=(71 \mathrm{~nm}$, $60 \mathrm{~nm})$ because a phase change of $2 \pi$ occurs around these points. $(\mathbf{b}) r_{b}=0$ at $\mathbf{P}_{1}^{\mathrm{T}}=(32 \mathrm{~nm}, 47 \mathrm{~nm})$ and $\mathbf{P}_{2}^{\mathrm{T}}=(60 \mathrm{~nm}, 71 \mathrm{~nm})$, where the first and second numbers correspond to $d_{1}$ and $d_{2}$, respectively.

\section{Results and Discussion}

In order to confirm one-way zero reflection, we calculated the reflectance of the simulated structures: $\mathrm{SiO}_{2}(32 \mathrm{~nm})-\mathrm{Au}(10 \mathrm{~nm})-\mathrm{SiO}_{2}(47 \mathrm{~nm})$ and $\mathrm{SiO}_{2}(60 \mathrm{~nm})-\mathrm{Au}(10 \mathrm{~nm})-$ $\mathrm{SiO}_{2}(71 \mathrm{~nm})$. Reflectance is defined as the square of the absolute value of the reflection coefficient. Therefore, with forwardly incident light the reflectance is $\left|r_{f}\right|^{2}$, for backwardly incident light it is $\left|r_{b}\right|^{2}$. Figure 3a shows the reflectance as a function of wavelength for $d_{1}=32 \mathrm{~nm}$ and $d_{2}=47 \mathrm{~nm}$. The blue solid curve is for the reflectance of forwardly incident light, the black dashed curve is for the reflectance of backwardly incident light. The numerically calculated reflectance is less than $10^{-5}$ for forwardly incident light while it is not zero but $2.5 \%$ for backwardly incident light at $\lambda=560 \mathrm{~nm}$. These results confirm that we have one-way zero reflection at $\lambda=560 \mathrm{~nm}$, which agrees with our simulation results presented in Figure2. Figure 3b shows the transmittances of $\left|t_{f}\right|^{2}\left(\left|t_{b}\right|^{2}\right)$ for forwardly (backwardly) incident light. The blue solid curve shows the transmittance of forwardly incident light; the black dashed curve shows the transmittance of backwardly incident light. The blue solid and black dashed curves overlap exactly, which means the transmittance for forwardly incident light is the same as for backwardly incident light.

(a)

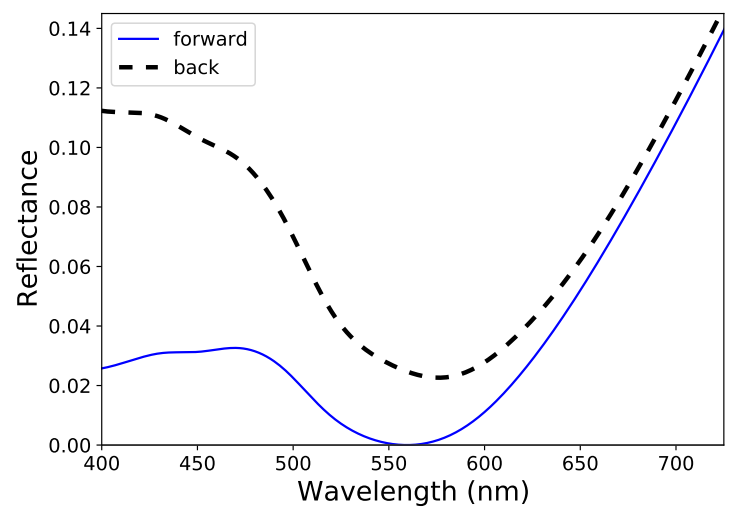

(b)

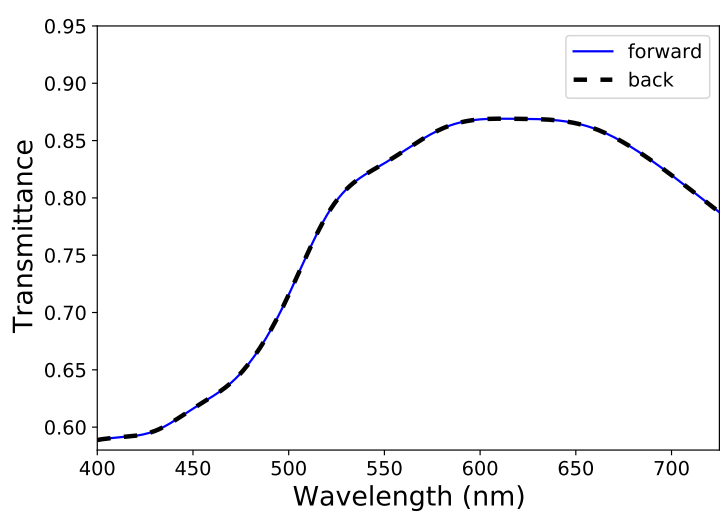

Figure 3. Reflectance and transmittance for a structure with $d_{1}=32 \mathrm{~nm}$ and $d_{2}=47 \mathrm{~nm}$ (a) Reflectance of forwardly incident light (blue solid curve) and backwardly incident light (black dashed curve) as a function of wavelength. Only reflectance of forwardly incident light becomes zero at $\lambda=560 \mathrm{~nm}$. (b) Transmittance of forwardly incident light (blue solid curve) and backwardly incident light (black dashed curve) as a function of wavelength. The transmittance is the same for both forwardly and backwardly incident light. 
Figure 4a shows the reflectance as a function of wavelength for $d_{1}=60 \mathrm{~nm}$ and $d_{2}=71 \mathrm{~nm}$. The blue solid curve shows the reflectance of forwardly incident light; the black dashed curve shows the reflectance of backwardly incident light. The numerically calculated reflectance is less that $10^{-5}$ for forwardly incident light while it is not zero but $1.4 \%$ for backwardly incident light at $\lambda=560 \mathrm{~nm}$. These results confirm that we achieve one-way zero reflection at $\lambda=560 \mathrm{~nm}$. Figure $4 \mathrm{~b}$ shows the transmittance. The blue solid curve shows the transmittance of forwardly incident light, the black dashed curve shows the transmittance of backwardly incident light. The blue solid and black dashed curves overlaps exactly.

The reflectance and transmittance results indicate that the absorption is different for forwardly and backwardly incident light because Reflectance + Transmittance + Absorption $=1$. Figure 5 shows the absorption spectra of forwardly incident light (blue solid curve) and backwardly incident light (black dashed curve) as a function of wavelength for $d_{1}=47 \mathrm{~nm}$ and $d_{2}=32 \mathrm{~nm}(\mathrm{a}), d_{1}=60 \mathrm{~nm}$ and $d_{2}=71 \mathrm{~nm}(\mathrm{~b})$. In both cases, absorption is higher for forwardly incident light compared to backwardly incident light.

(a)

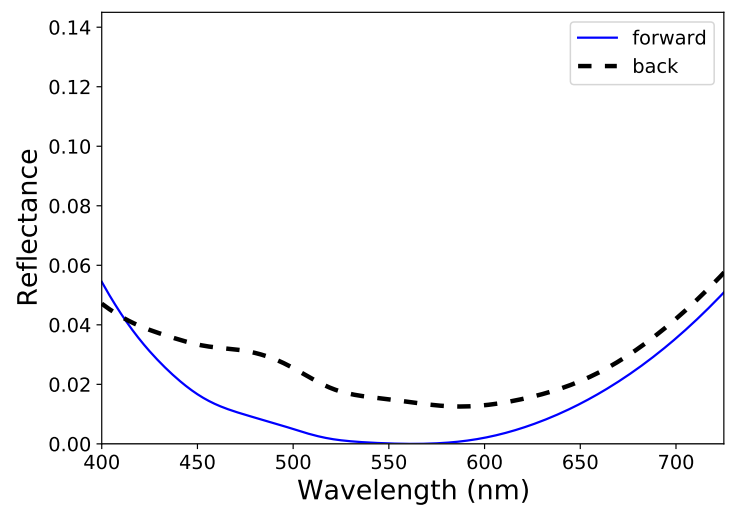

(b)

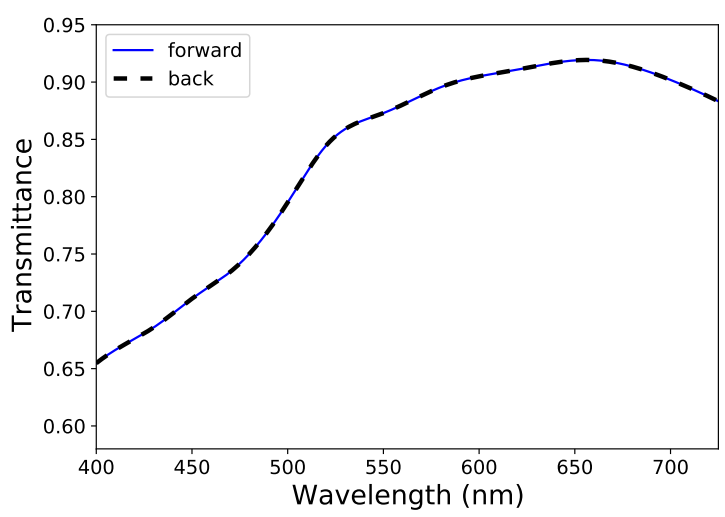

Figure 4. Reflectance and transmittance for a structure with $d_{1}=60 \mathrm{~nm}$ and $d_{2}=71 \mathrm{~nm}$ (a) Reflectance of forwardly incident light (blue solid curve) and backwardly incident light (black dashed curve) as a function of wavelength. Only the reflectance of forwardly incident light becomes zero at $\lambda=560 \mathrm{~nm}$. (b) Transmittance of forwardly incident light (blue solid curve) and backwardly incident light (black dashed curve) as a function of wavelength. The transmittance is the same for both forwardly and backwardly incident light.

(a)

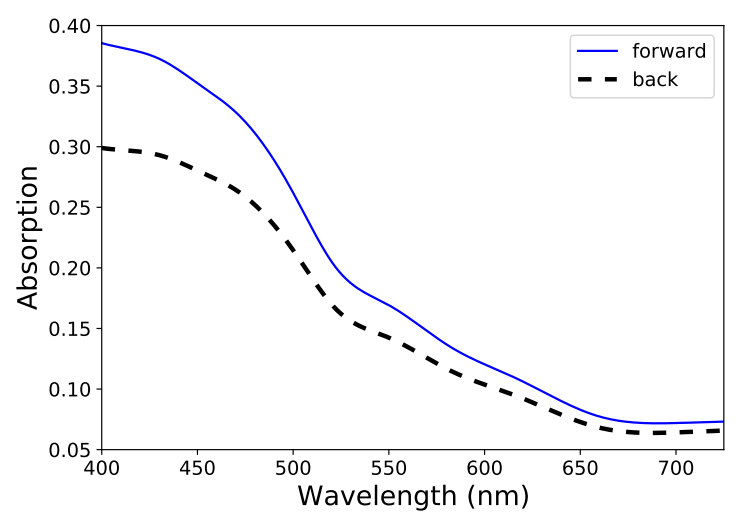

(b)

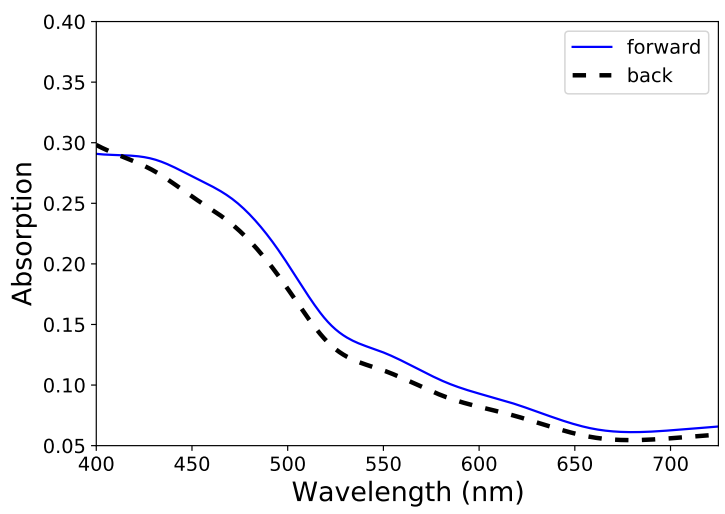

Figure 5. Absorption spectra of forwardly incident light (blue solid curve) and backwardly incident light (black dashed curve) as a function of wavelength for a structure with $d_{1}=47 \mathrm{~nm}$ and $d_{2}=32 \mathrm{~nm}(\mathbf{a}), d_{1}=60 \mathrm{~nm}$ and $d_{2}=71 \mathrm{~nm}(\mathbf{b})$. 


\section{Conclusions}

We used TMM to simulate one-way zero reflection through an insulator-metal-insulator (IMI) structure. We used $\mathrm{SiO}_{2}$ for the two outer insulator layers and $\mathrm{Au}$ for the middle metal layer. By examining a range of thicknesses for both the $\mathrm{SiO}_{2}$ layers with the 10-nm Au layer at $\lambda=560 \mathrm{~nm}$, we found two pairs of thicknesses that produced one-way zero reflection: $d_{1}=47 \mathrm{~nm}(60 \mathrm{~nm})$ for the top layer and $d_{2}=32 \mathrm{~nm}(71 \mathrm{~nm})$ for the bottom layer. We also confirmed one-way zero reflection using reflectance spectra. The reflectance becomes zero for forwardly incident light, while it is not zero for backwardly incident light. The reflectance of backwardly incident light is higher when $d_{1}=47 \mathrm{~nm}$ and $d_{2}=32 \mathrm{~nm}$ compared to when $d_{1}=60 \mathrm{~nm}$ and $d_{2}=71 \mathrm{~nm}$.

Even though we used a metal as the absorption material, any material with absorption could be used to achieve one-way zero reflection, such as silicon demonstrated in prior research with various configurations [16,17]. IMI structure proposed in the context of one-way zero reflection may be adapted in optical fiber-based biosensors to investigate the possible increase of light coupling to the sensing region [23]. One-way zero reflection has great potential when applied in systems where no reflection is important, such as in solar cells.

Author Contributions: Conceptualization, H.N. and J.-M.C.; methodology, H.N.; investigation, H.N.; data curation, J.-M.C.; writing—original draft preparation, H.N.; writing—review and editing, J.-M.C.; visualization, J.-M.C.; supervision, H.N.; project administration, H.N.; funding acquisition, H.N. All authors have read and agreed to the published version of the manuscript.

Funding: This work was supported by the Basic Science Research Program through the National Research Foundation of Korea (NRF) funded by the Ministry of Education (No. 2017R1D1A1A02018515).

Institutional Review Board Statement: Not applicable.

Informed Consent Statement: Not applicable.

Conflicts of Interest: The authors declare no conflict of interest.

\section{References}

1. Bender, C.M.; Boettcher, S. Real Spectra in Non-Hermitian Hamiltonians Having PT Symmetry. Phys. Rev. Lett. 1998, 80, 5243-5246. [CrossRef]

2. Longhi, S.; Della Valle, G. Photonic Realization of PT-Symmetric Quantum Field Theories. Phys. Rev. A 2012, 85, 012112. [CrossRef]

3. Bender, C.M. Making Sense of Non-Hermitian Hamiltonians. Rep. Prog. Phys. 2007, 70, 947-1018. [CrossRef]

4. Musslimani, Z.H.; Makris, K.G.; El-Ganainy, R.; Christodoulides, D.N. Optical Solitons in PT Periodic Potentials. Phys. Rev. Lett. 2008, 100, 030402. [CrossRef] [PubMed]

5. Guo, A.; Salamo, G. J.; Duchesne, D.; Morandotti, R.; Volatier-Ravat, M.; Aimez, V.; Siviloglou, G.A.; Christodoulides, D.N. Observation of PT-Symmetry Breaking in Complex Optical Potentials. Phys. Rev. Lett. 2009, 103, 093902. [CrossRef] [PubMed]

6. Rüter, C.E.; Makris, K.G.; El-Ganainy, R.; Christodoulides, D.N.; Segev, M.; Kip, D. Observation of Parity-Time Symmetry in Optics. Nat. Phys. 2010, 6, 192-195. [CrossRef]

7. Miri, M.-A.; LiKamWa, P.; Christodoulides, D.N. Large Area Single-Mode Parity-Time-Symmetric Laser Amplifiers. Opt. Lett. 2012, 37, 764. [CrossRef]

8. Lin, Z.; Ramezani, H.; Eichelkraut, T.; Kottos, T.; Cao, H.; Christodoulides, D.N. Unidirectional Invisibility Induced by PTSymmetric Periodic Structures. Phys. Rev. Lett. 2011, 106, 213901. [CrossRef]

9. $\quad$ Liertzer, M.; Ge, L.; Cerjan, A.; Stone, A.D.; Türeci, H.E.; Rotter, S. Pump-Induced Exceptional Points in Lasers. Phys. Rev. Lett. 2012, 108, 173901. [CrossRef]

10. Feng, L.; Xu, Y.L.; Fegadolli, W.S.; Lu, M.H.; Oliveira, J.E.B.; Almeida, V.R.; Chen, Y.F.; Scherer, A. Experimental Demonstration of a Unidirectional Reflectionless Parity-Time Metamaterial at Optical Frequencies. Nat. Mater. 2013, 12, 108-113. [CrossRef]

11. Brandstetter, M.; Liertzer, M.; Deutsch, C.; Klang, P.; Schöberl, J.; Türeci, H.E.; Strasser, G.; Unterrainer, K.; Rotter, S. Reversing the Pump Dependence of a Laser at an Exceptional Point. Nat. Commun. 2014, 5, 4034. [CrossRef] [PubMed]

12. Feng, L.; Wong, Z.J.; Ma, R.M.; Wang, Y.; Zhang, X. Single-Mode Laser by Parity-Time Symmetry Breaking. Science 2014, 346, 972-975. [CrossRef] [PubMed]

13. Hodaei, H.; Miri, M.A.; Hassan, A.U.; Hayenga, W.E.; Heinrich, M.; Christodoulides, D.N.; Khajavikhan, M. Parity-TimeSymmetric Coupled Microring Lasers Operating around an Exceptional Point. Opt. Lett. 2015, 40, 4955. [CrossRef] [PubMed]

14. Miri, M.-A.; Alú, A. Exceptional points in optics and photonics. Science 2019, 363, eaar7709. [CrossRef]

15. Wiersig, J. Review of exceptional point-based sensors. Photonics Res. 2020, 8, 1457-1467. [CrossRef] 
16. Shen, Y.; Deng, X.H.; Chen, L. Unidirectional Invisibility in a Two-Layer Non-PT-Symmetric Slab. Opt. Express 2014, $22,19440$. [CrossRef]

17. Feng, L.; Zhu, X.; Yang, S.; Zhu, H.; Zhang, P.; Yin, X.; Wang, Y.; Zhang, X. Demonstration of a Large-Scale Optical Exceptional Point Structure. Opt. Express 2014, 22, 1760. [CrossRef]

18. Huang, Y.; Veronis, G.; Min, C. Unidirectional Reflectionless Propagation in Plasmonic Waveguide-Cavity Systems at Exceptional Points. Opt. Express 2015, 23, 29882. [CrossRef]

19. Na, J.; Noh, H. Investigation of a Broadband Coherent Perfect Absorber in a Multi-Layer Structure by Using the Transfer Matrix Method. J. Korean Phys. Soc. 2018, 72, 66-70. [CrossRef]

20. Ducourtieux, S.; Podolskiy, V.A.; Grésillon, S.; Buil, S.; Berini, B.; Gadenne, P.; Boccara, A.C.; Rivoal, J.C.; Bragg, W.D.; Banerjee, K.; et al. Near-Field Optical Studies of Semicontinuous Metal Films. Phys. Rev. B 2001, 64, 1654031-16540314. [CrossRef]

21. Johnson, P.B.; Christy, R.W. Optical Constants of the Noble Metals. Phys. Rev. B 1972, 6, 4370-4379. [CrossRef]

22. Andreasen, J.; Vanneste, C.; Ge, L.; Cao, H. Effects of Spatially Nonuniform Gain on Lasing Modes in Weakly Scattering Random Systems. Phys. Rev. A 2010, 81, 043818. [CrossRef]

23. González-Vila, Á.; Debliquy, M.; Lahem, D.; Zhang C.; Mégret, P.; Caucheteur, C. Molecularly imprinted electropolymerization on a metal-coated optical fiber for gas sensing application. Sens. Actuators B Chem. 2017, 244, 1145-1151. [CrossRef] 